\title{
Hardware and Software System for Thermal Control
}

\author{
Yu. Yu. Gromov ${ }^{1, *}$, A. A. Churikov ${ }^{1}$, A. G. Divin ${ }^{1}$, I. N. Ishchuk ${ }^{2}$, S.A. Barkalov ${ }^{2}$ \\ ${ }^{1}$ Tambov State Technical University, Institute of Automation and Information Technology, Tambov, Russia, \\ ${ }^{2}$ Zhukovsky-Gagarin Air Force Academy, Meteorology Department, Voronezh, Russia
}

Received 5 October 2016; Accepted 24 December 2016

\begin{abstract}
The transition from a one-dimensional analysis of the thermal process in the flat sample to the analysis of rather complex thermal process in a cylindrical sample, as close as possible to the actual product. This resulted in the development of the mathematical apparatus for solving multidimensional problems of heat conduction and creation of hardware and software thermal control.
\end{abstract}

Keywords: thermal control, problem of heat conduction, temporal integral characteristic, surface-temporal integral characteristic

\section{Introduction}

In the mid 60 -ies the 1 st Research Institute, headed by A. Nadiradze (Moscow Institute of Thermal Technology), started elaborating the project of mobile basing solid-fuel ICBMs by using the experience of the creation in 1965 the operational-tactical Temp-S missile. In design engineering bureau located in Hot'kovo near Moscow there were developed a fiberglass engine body and transporter-launcher container (later of carbon materials) and adjusted their production. The whole period of operation the missile itself was in a sealed TLC (transporter-launcher container), made of fiberglass with a thick layer of heat protective material. The breach of a heat-shielding properties led to a trouble in the rocket design geometry, and, consequently, to a disorder of the possible flight trajectory and project mission performance.

Methods of thermal control as insulating the large diameter cylindrical shape products and the analysis of products thermal storage modes in heat-insulating constructions of rather large products, have shown that the presence of even a local (point) external insulation defect or changing the insulation material heat-shielding properties is not local (point), as in the case of flat-dimensional products but "cylindrical" encompasses an annular domain of isolation and product. This can cause significant disturbance of geometric parameters of the product in the container (vertical, horizontal, displacement angle, etc.). Therefore, we first moved from a one-dimensional analysis of the thermal process in the flat sample to the analysis of rather complex thermal process in a cylindrical sample, as close as possible to the actual product. This resulted in the development of the mathematical apparatus for solving multidimensional problems of heat conduction and creation of hardware and software thermal control (TC).

This thermal control (TC) complex is one of the main components of the project to create a complex system of diagnostics thermal properties. Previously, the method and the measuring mobile probe of TC was used to control the properties of external insulation elements of the space shuttle "Buran", and was later supplemented by means of measurement for the diagnosis of substances in liquid phase for their thermal and rheological properties. The solving of the problem of heat loss minimizing for objects producing, transmitting or using thermal energy is possible with the use of this complex.

\section{Method and measuring device for monitoring TPP products from solid materials}

To determine the quality of solid products it is necessary to measure thermo- physical properties (TPP) in several areas subjected to damage and the loss of thermal properties, for example, in areas close to the edge and the center. As a rule, TPP reading considers a hand labor, and the man performs a monotonous job for a long time, which affects his fatigability and reduces the work efficiency on the whole. Therefore, our task is to develop rapid methods to control the product properties of horizontal and vertical surface area and automatize this process. Thus, for studying the thermal properties of the product surface area most suitable for us, in terms of operability, are information-measuring systems (IMS) that implement pulse methods making it possible to carry out non-destructive express control of solid materials (NC TPP).

Consider the non-destructive control of thermal properties of cylindrical shape solid products with a large radius of curvature. Hereafter the possibility of nondestructive control of thermal conductivity $\lambda$ and thermometric conductivity $\alpha$ of heat-protective solid material products with cylindrical surface are examined (Fig. 1). The result of this section is the confirmation of the concept of TPS control generalized method presented in this article. 


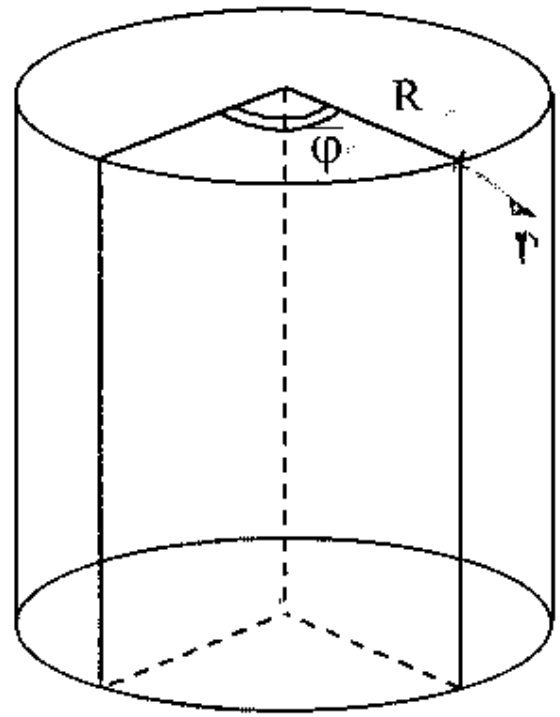

Fig. 1 - Physical model of the test work-piece

Depending on the type of information about the thermal effect on the tested body, consider the solution of inverse heat conduction problem, i.e. the problem of determining the values of thermal constants $\alpha$ and $\lambda$ for the following two cases:

it is known the heat flux density $q$ directed to the investigated cylindrical body with $R$ radius through the area of the side surface as a strip located between the two generators with the arc angle $2 \bar{\phi}$ between them;

it is known a power of the heat source as an endless strip located in the contact plane of two bodies: a cylinder surrounded over the side surface by unbounded body. At the thermal properties $a_{0}$ and $\lambda_{0}$ of the upper body is assumed to be known and the cylinder $a$ and $\lambda$ have to be determined. The tested bodies are considered to be isotropic and their thermal properties do not depend on the temperature that is quite possible, as an excessive temperature when realize the experiment for determination the thermal properties is less than $15 \mathrm{~K}$.

For the first case the thermal process in the tested body is described by the following boundary value problems of heat conduction:

$$
\frac{\partial U(r, \phi, t)}{\partial t}=\mathrm{a}\left(\frac{\partial^{2} U(r, \phi, t)}{\partial r^{2}}+\frac{1}{r} \frac{\partial U(r, \phi, t)}{\partial r}+\frac{1}{r} \frac{\partial^{2} U(r, \phi, t)}{\partial \phi^{2}}\right),
$$$$
(t>0,0 \leq r \leq R, 0 \leq \phi \leq 2 \pi) \text {; }
$$

$U(r, \phi, 0)=0$

$$
\frac{\partial U(r, \phi, t)}{\partial r}=\left\{\begin{array}{cc}
q(\phi, t) / \lambda & r=R,|\phi| \leq \bar{\phi} \\
0 & r=R,|\phi|>\bar{\phi}
\end{array}\right.
$$

$\left.\frac{\partial U(r, \phi, t)}{\partial \phi}\right|_{\substack{\phi=0 \\ \phi=\pi}}=0 ; U(0, \phi, t)<\infty$.
Because of the symmetry of temperature distribution in the cylinder relatively to the planes $\phi=0$ and $\phi=\pi$ consider the solution of the problem for domain of variation $\phi \in[0, \pi]$. The problem solution (1) - (4) in the Laplace image area and finite integral Fourier cosine transformation within the range of $[0, \pi]$ for the surface temperature of the cylinder $r=R$ is given by

$U_{c}^{*}(R, v, p) \equiv U_{c}^{*}(v, p)=\frac{q_{c}^{*}(v, p) R}{\lambda \Phi(g)}$,

where $g=R \sqrt{p / \mathrm{a}}$;

$\Phi(g)=\left[g I_{v+1}(g)\right] /\left[I_{v}(g)+v\right]$

$I_{v}(g)$ - modified Bessel function of the first kind of $v$ order; $p$ - the Laplace transformation parameter $p>0 ; v$ the Fourier transformation parameter to the variable $\phi$ when $v \geq 0$.

The solution (5) in the Laplace image area the equation takes the form of

$U^{*}(\phi, p)=\frac{2 R}{\lambda \pi} \sum_{v=0}^{\infty} \frac{q_{c}^{*}(v, p) \cos (v \phi)}{\beta_{v} \Phi(g)}$,

where

$$
\beta_{v}=\left\{\begin{array}{cc}
2 & v=0 \\
1 & v \neq 0
\end{array}\right.
$$

Temperature image or heat flux of type

$F^{*}(p)=\int_{0}^{\infty} \exp (-p t) f(t) d t \quad$ at $\quad p>0$

name as a temporal integral characteristic (TIC) of temperature or heat flux, and the image $F_{c}^{*}(v, p)=\int_{0}^{\pi} \cos (v \phi) \int_{0}^{\infty} \exp (-p t) f(\phi, t) d \phi d t$, when $v>0$, $p>0$ as a surface-temporal integral characteristic (STIC) of temperature or heat flux.

We show the possibility of determining the values of thermal diffusivity of the tested body by using formulas (5) and (6) subject to TIC and STIC temperature and heat flow we can calculate with the experimental values.

Assume that we know: STIC $U_{c}^{*}(v, p)$ and heat flow $q_{c}^{*}(v, p)$ for two values $p_{1}$ and $p_{2}$ of the parameter $p$ and the numerical value of the parameter $v$, i.e. we know $U_{c}^{*}\left(v, p_{2}\right), U_{c}^{*}\left(v, p_{1}\right), q_{c}^{*}\left(v, p_{2}\right), q_{c}^{*}\left(v, p_{1}\right)$. Then, using the ratio of STIC temperature (5) at two values $p_{1}$ and $p_{2}$ we get the following equation to determine subtended expressed therein the value of thermal conductivity a : 


$$
\frac{U_{c}^{*}\left(v, p_{1}\right)}{U_{c}^{*}\left(v, p_{2}\right)}=\frac{q_{c}^{*}\left(v, p_{1}\right)}{q_{c}^{*}\left(v, p_{2}\right)} \cdot \frac{\Phi\left(g_{2}\right)}{\Phi\left(g_{1}\right)}
$$

where $g_{i}=R \sqrt{p_{i} / \mathrm{a}}, i=1,2$.

The calculation of thermal conductivity $\lambda$ is carried out on the following equation: $\lambda=\frac{q_{c}^{*}\left(v, p_{i}\right) R}{U_{c}^{*}\left(v, p_{i}\right) \Phi\left(g_{i}\right)}, \quad i=1,2$ where $g_{i}$ found in (8) and $\Phi\left(g_{i}\right)$ corresponds to (6).

If the heat flux supplied to the tested body surface within the heating area does not depend on the coordinate $\phi$ then the value of the TIC temperature (7) is defined by the following formula:

$$
U^{*}(\phi, p)=\frac{q^{*}(p) R}{\lambda} V(\phi, g)
$$

where

$$
V(\phi, g)=\frac{2}{\pi} \sum_{v=0}^{\infty} \frac{\sin (v \bar{\phi}) \cos (v \phi)}{v \beta_{v} \Phi(g)} .
$$

The determination of values $a$ and $\lambda$, using equation (9) can be done in two ways.

For the first method we use value of TIC temperature in two arbitrary points $\phi_{1}$ and $\phi_{2}$ for a certain parameter $p$. Then the calculation of the thermal conductivity and diffusivity we provide by the following equations:

$\frac{U^{*}\left(\phi_{1}, p\right)}{U^{*}\left(\phi_{2}, p\right)}=\frac{V\left(\phi_{1}, g\right)}{V\left(\phi_{2}, g\right)}, \lambda=\frac{q^{*}(p) R}{U^{*}\left(\phi_{i}, p\right)} V\left(\phi_{i}, g\right), \quad i=1,2$.

By the second method we use the value of TIC temperature $U^{*}(\phi, p)$ at one point $\phi=0$ at two different values $p_{1}$ and $p_{2}$ of $p$ parameter. We get the following formulas for calculation $\mathrm{a}$ and $\lambda$ :

$$
\frac{U^{*}\left(\phi, p_{1}\right)}{U^{*}\left(\phi, p_{2}\right)} \cdot \frac{q^{*}\left(p_{2}\right)}{q^{*}\left(p_{1}\right)}=\frac{V\left(g_{1}\right)}{V\left(g_{2}\right)}, \quad \lambda=\frac{q^{*}\left(p_{i}\right) R}{U^{*}\left(\phi, p_{i}\right)} V\left(g_{i}\right),
$$

where

$$
V(g)=\frac{2}{\pi} \sum_{v=0}^{\infty} \frac{\sin (v \bar{\phi})}{v \beta_{v} \Phi(g)} ; g_{i}=R \sqrt{p_{i} / \mathrm{a}}, i=1,2
$$

For the second case, i.e. for the two-body system, heat distribution process is described by the following boundary value problem of heat conductivity:

$$
\frac{\partial U_{0}(r, \phi, t)}{\partial t}=\mathrm{a}_{0}\left(\frac{\partial^{2} U_{0}(r, \phi, t)}{\partial r^{2}}+\frac{\partial U_{0}(r, \phi, t)}{r \partial r}+\frac{\partial^{2} U_{0}(r, \phi, t)}{r \partial \phi^{2}}\right)
$$

$(0 \leq r \leq R, 0 \leq \phi \leq 2 \pi, t>0)$

$$
\frac{\partial U(r, \phi, t)}{\partial t}=\mathrm{a}\left(\frac{\partial^{2} U(r, \phi, t)}{\partial r^{2}}+\frac{1}{r} \frac{\partial U(r, \phi, t)}{\partial r}+\frac{1}{r} \frac{\partial^{2} U(r, \phi, t)}{\partial \phi^{2}}\right),
$$

$(R \leq r<\infty, 0 \leq \phi \leq 2 \pi, t>0)$

$$
U_{0}(r, \phi, 0)=U(r, \phi, 0)=0
$$

$\left.\frac{\partial U_{0}(r, \phi, t)}{\partial \phi}\right|_{\phi=0}=\left.\frac{\partial U(r, \phi, t)}{\partial \phi}\right|_{\phi=0}=0 ;$

$\lambda \frac{\partial U(r, \phi, t)}{\partial r}-\lambda_{0} \frac{\partial U_{0}(r, \phi, t)}{\partial r}=$

$=\left\{\begin{array}{cc}Q(\phi, t) & r=R,|\phi| \leq \bar{\phi}, \\ 0 & r=R,|\phi|>\bar{\phi}\end{array}\right.$

$U_{0}(R, \phi, t)=U(R, \phi, t)$

$U_{0}(r, \phi, t) \rightarrow 0$ at $r \rightarrow \infty ; U(0, \phi, t)<\infty$.

where $Q(\phi, t)$ is the power source, acting in the plane of two bodies contact. The solution of problems (10) - (16) as a STIC temperature for cylinder surface $r=R$ has the form

$$
U_{c}^{*}(R, v, p) \equiv U_{c}^{*}(v, p)=\frac{Q_{c}^{*}(v, p) R}{\lambda \Phi(g)+\lambda_{0} L\left(q_{0}\right)},
$$

where

$$
\begin{aligned}
& L\left(g_{0}\right)=g_{0} K_{v+1}\left(g_{0}\right) / K_{v}\left(g_{0}\right)+v ; \\
& g_{0}=R \sqrt{p / \mathrm{a}_{0}} ; \\
& K_{v}(x)
\end{aligned}
$$

a modified Bessel function of the second kind of $v$ order. If you know the value of temperature integrated characteristics $U_{c}^{*}(v, p)$ for two values of the $p$ parameter: $p_{1}=p$ and $p_{2}=k p$ when $k=$ const and for some value of the $v$ parameter, i.e. $U_{c}^{*}\left(v, p_{1}\right), U_{c}^{*}\left(v, p_{2}\right)$, then the value of the thermal diffusivity $a$ may be determined from the following equation:

$$
\frac{Q_{c}^{*}\left(v, p_{1}\right) / U_{c}^{*}\left(v, p_{1}\right)-\lambda_{0} L\left(g_{01}\right) / R}{Q_{c}^{*}\left(v, p_{2}\right) / U_{c}^{*}\left(v, p_{2}\right)-\lambda_{0} L\left(g_{02}\right) / R}=F(k, g)
$$

where

$$
g_{0 i}=R \sqrt{p_{i} / \mathrm{a}_{0}}, i=1,2 ; F(k, g)=\frac{\Phi(g)}{\Phi(g \sqrt{k})} ;
$$


and the thermal conductivity

$$
\lambda=\frac{Q_{c}^{*}\left(v, p_{i}\right) R / U_{c}^{*}\left(v, p_{i}\right)-\lambda_{0} L\left(g_{0 i}\right)}{\Phi\left(g_{i}\right)}, \quad i=1,2 .
$$

Assume that the thermal process in a given system of bodies takes place under the following additional conditions:

a) in the plane of two bodies contact the heat transfer between them is neglectfully small and heat flow with density $q_{0}(t)$ and $q(t)$, directed into each of the bodies from a source of heat with $Q$ power are determined by the equation $q_{0}(t)+q(t)=Q$;

b) on the central axis of the heater, i.e. at $r=R$ and $\phi=0$ the first body temperature is equal to the second body temperature $U_{0}(R, 0, t)=U(R, 0, t)$. Then, TIC temperature of the upper and lower body at $r=R, \phi=0$ will be respectively as follows:

$U_{0}^{*}(R, 0, p) \equiv U_{0}^{*}(p)=\frac{q_{0}^{*}(p) R}{\lambda_{0}} N\left(g_{0}\right)$

$U^{*}(R, 0, p) \equiv U^{*}(p)=\frac{q^{*}(p) R}{\lambda} V(g)$,

where

$N\left(g_{0}\right)=\frac{2}{\pi} \sum_{v=0}^{\infty} \frac{\sin (v \bar{\phi})}{v \beta_{v} L\left(g_{0}\right)}$

Using two values $p_{1}$ и $p_{2}$ and $p$ parameter the values of TIC temperature $U_{0}^{*}(p)$ и $U^{*}(p)$ and the heater $Q$ power, we get the following dependence to determine the unknown quantities $a$ and $\lambda$ :

$\left[\frac{Q}{p_{2} U^{*}\left(p_{2}\right)}-\frac{\lambda_{0}}{R N\left(g_{02}\right)}\right] /\left[\frac{Q}{p_{1} U^{*}\left(p_{1}\right)}-\frac{\lambda_{0}}{R N\left(g_{01}\right)}\right]=W(k, g)$,

$\lambda=\left[\frac{Q R}{p_{i} U^{*}\left(p_{i}\right)}-\frac{\lambda_{0}}{N\left(g_{0 i}\right)}\right] V\left(g_{i}\right)$,

where $W(k, g)=\frac{V(g)}{V(g \sqrt{k})}$

To calculate the thermo-physical coefficients by formulas (17) and (18) we use the functions $V(g)$ and $W(k, g)$ that are calculated in advance for the whole range of changing the $g$ parameter in this experiment. The value of $W(k, g)$ function is calculated according to experimental data $U^{*}\left(p_{2}\right), U^{*}\left(p_{1}\right)$ and $Q$ by formula (17). Using the value of $W(k, g)$ function, we find the $g$ parameter by which, in its turn, we determine the value of the function $V(g)$ and value of the thermal diffusivity a :

$\mathrm{a}=p R^{2} / g^{2}$

and then $\lambda$ by the formula (18).

Using in practice while investigating of TPP materials obtained formulas depends on the desired accuracy of TPP determination and is determined by the capabilities of appropriate circuitry technical implementation both for setting the required heat fluxes and the ways of measuring the temperature.

Note that TIC temperature is calculated by measuring the temperature at one point, but STIC is in several points of tested body surface. The theoretical analysis has shown that the use of STIC in comparison with TIC temperature leads to reduction of the TPP inaccuracy determining, but the measuring circuit, the preparation and conduction of the experiment is complicated. Structurally, the substrate is fixed in a case with the help of a metal support 3 (Fig. 2) which, in its turn, is attached inside the probe via push clips, which allows to replace the sensor quickly and easily, i.e. the substrate is fixed in the case by simple click of 4 buttonscolumns of 6 support in the slots 7 .

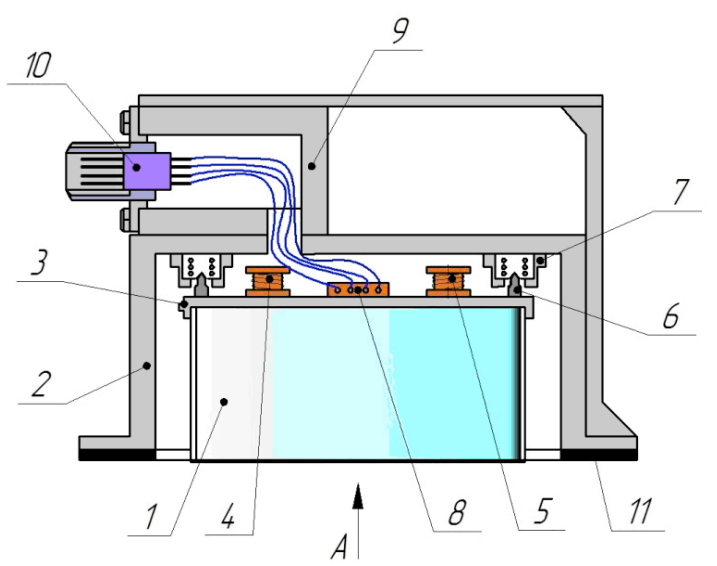

Image A

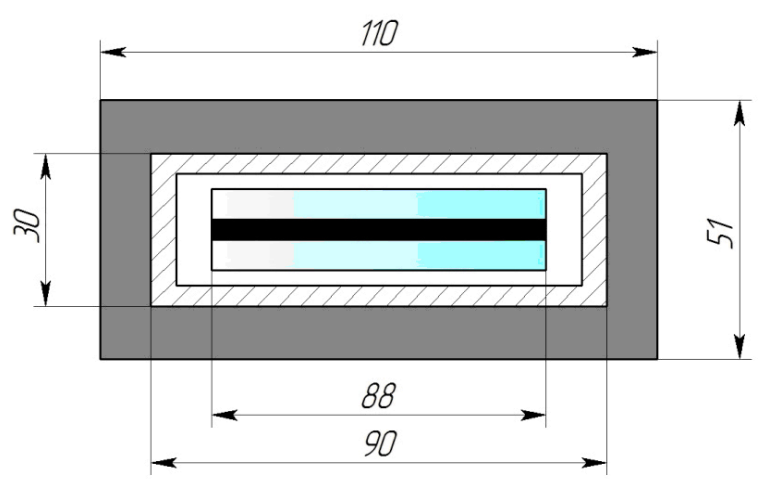

(Probe bottom is shaded)

Fig. 2 - Measuring device for control the thermo physical characteristics of cylindrically shaped large radius products from solid materials 
A probe case is made as a hollow rectangular parallelepiped with dimensions of $(40 \times 50 \times 90) \mathrm{mm}^{3}$, opened from one side. Inside the probe a chock from the reference material is mounted on elastic springs, on the outside of which a flat heater made of constantan wire is attached. The thermoelectric converter with a nominal static characteristic (NSC) XA(K) is placed on the central axis of the heater. The thermocouple operating junction is located in the center of the heater. Its leads from the ends of the heater are allocated to the cold junction coils 4 and 5. The thermo electrodes are welded end-to-end, and this made it possible to place them between the wires of the electric heater without any contact with it. From the cold junction coils 4 and 5, already with copper wires, the thermocouple via the 8 and 10 connector is connected with an external apparatus. The case of the 2 probe is a hollow parallelepiped made of metal. The 1 substrate made of a reference material is attached inside a probe on a special platform. Flat heater made of constantan wire is glued with temperature resistance adhesive on the substrate.

The heater is made thinned, i.e. so that each of its separate wires on the heater (wire is much more than in tested materials) and the temperature field under real heater does not violate. Its connections from the ends of the heater are assigned to the cold junction coils 4 and 5 (Fig. 2).

Lower substrate base is fixed to the base 3 , on the inner side of which the cold junction coils 4, 5 are attached and the connector 8 for connecting with a thermocouple with measuring unit of a device and a heater with power supply. Four supports 6 (Fig. 3) are formed so that they can easily enter the slots 7. This makes it possible to change the substrate quickly from experiment to experiment.

At the edge of the open side of the probe it is welded a wide flange to which is glued a rubber gasket 11 , which excludes the sliding on the sample surface. A load $(\approx 14 \mathrm{~kg})$ which provides a tight standard compression, and also the heater to the tested surface rests on edge. Tight held reduces the contact thermal resistance and therefore reduces error in determining the thermo-physical characteristics of the materials. For convenience, the probe has a handle 12, attached to the upper bottom of the probe on the outside.

\section{Measuring device for monitoring fluids TPP}

To control the thermo-physical properties of the fluids it is used a measuring device on the basis of two coaxial cylinders (see. Fig.3). Inner cylinder of this measuring instrument is made of heat-resistant material (Polyether ether ketone (PEEK)), can withstand prolonged exposure of a temperature to $200{ }^{\circ} \mathrm{C}$ and maintaining at this its mechanical properties and chemical composition.

The working part of the cylinder 1 has two wire electrical heaters 4, 5 from the constant and two resistance temperature devices from a copper wire placed in close proximity to the heater coil. Inner coil 4 reserved sleeve 2, which is placed on top of the winding 5 .

On the run of thermo-physical experiment an electric current runs through heater coil 4 and 5 what causes the release of heat therein. Coil temperatures are supported equal by the power control supplied to the inner coil. Thus, heat flux, evolving by the heater 5 moves preferably to a protective sleeve layer 6 , then to the test liquid 3 and in the outer cylinder 7 . Therefore, the heater 4 may be assumed as protective.

A mathematical model of the temperature field in the measuring device layers after the heaters switching includes differential equations, initial, boundary and additional conditions. The inverse problem solution allows defining the thermo-physical characteristics of the test liquid.

Electrical schematic diagram of the measurement setup for determining the thermo-physical properties of fluid by coaxial cylinders method is presented in Fig. 4.

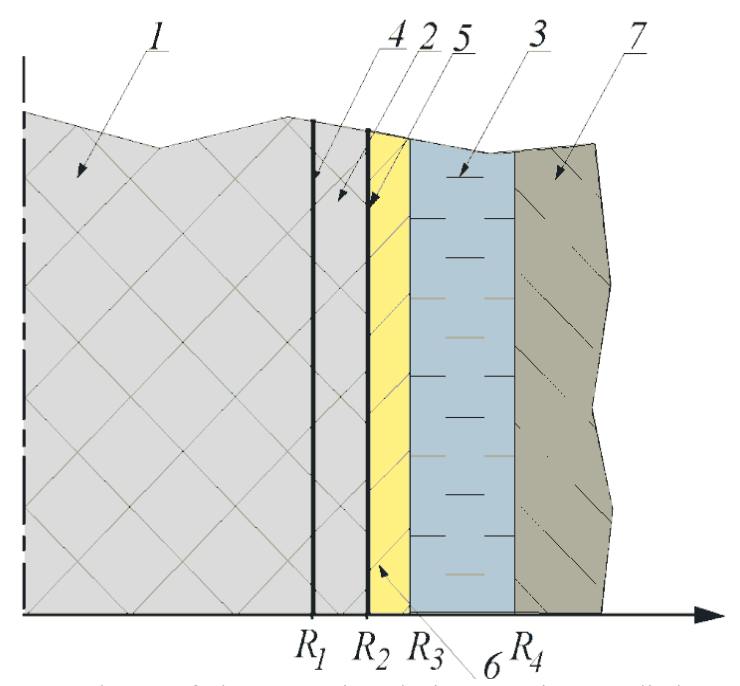

Fig. 3 - Scheme of the measuring device, 1 - inner cylinder, 2 protective sleeve, 3 - test liquid, 4 - protective heater, 5 - main heater, 6 - protective sleeve, 7 - external (rotating) cylinder

Resistance temperature device $R K 2$ is included into the measuring bridge on the two-wire circuit. Bridge is powered by $H Y 3003 D-2$ power supply with voltage of $30 \mathrm{~V}$ across the ballast resistor $R 7$, which is in the heating mode, is shunted by the solid-state relay $A 7$ of $M-O D C 5$ type. The bridge output voltage is supplied to the channel $A I 01$ of $A 1$ data acquisition board PCI 6251 type on differential circuit. The digital equivalent of the unbalance voltage is converted in software of a personal computer (PC) processor to a temperature value. At the same time, in accordance with PID-law it is generated an output signal on the analog output $A O 01$ of card $A 1$, which controls the operation of the control unit triac and thyristor $(A 5)$. The output of this unit is a constant voltage in the range of $\left(\begin{array}{lll}0 & \ldots & 127\end{array}\right) \mathrm{V}$, which is supplied to the $E K$ heater of the measuring device (MD). Resistance temperature device $R K 1$, located in MD in the immediate vicinity of the EK heater, receives its temperature, which is a software PID controller maintains equal to the temperature of MD in place of $R K 2$ installation by changing the power supplied to the $E K$ heater. The general form of the measurement setup is presented in Fig. 5 .

Measurement of rotational moment transferred to the inner cylinder of measuring device by viscous friction forces in the layer of the test liquid is carried out by means of strain indicators $A 8$ and $A 9$, the first of which works in compression and the second in tension. The difference between the output voltages of the inverter is proportional to the rotational moment and depends little on the temperature changes. Transmitters are powered by voltage of $15 \mathrm{~V}$ from power supply unit of $A 10$, and their outputs are connected to the channels $A I 3$ and $A I 4$ of data acquisition card under the scheme with a common ground.

Thus, thermal conductivity, thermal diffusivity coefficient and heating capacity of the test fluid are determined by the temperature response to a thermal 
Yu.Yu. Gromov, A.A. Churikov, A.G. Divin, I.N. Ishchuk, S.A. Barkalov/

Journal of Engineering Science and Technology Review 9 (6) (2016) 135-141

influence from the $E K$ heater both when the outer cylinder rotates and is at rest. By varying the speed of cylinder rotation it is possible to determine the dependence of the thermo-physical characteristics of fluids on the sliding velocity in the range up to $0100 \mathrm{~s}^{-1}$. Indicating the rotational moment (transmitted to the outer cylinder by means of the test fluid viscous friction forces) as a function of the sliding velocity, it is possible to form a flow curve and identify with it the rheological properties both Newtonian and non-Newtonian fluids.

\section{Conclusion}

The efficiency of the automatic systems for control the thermo-physical properties of products made of solid material has been tested in TSTU laboratory conditions, under production-line conditions at factories SPC "Technology".

The testing was conducted on the samples with known thermal properties. The measuring device for determining the solid materials TPC was tested on bulk samples of polimethyl methacrylate, polystyrene, asbestos-reinforced laminate, fibre-glass laminate and other similar materials. Measurement accuracy of thermal conductivity and thermal diffusivity coefficients is $7 \%$ and of volume heat capacity is $10 \%$.

Measuring device for determining the fluids TPC was tested on distilled water and glycerin. According to the results of calibration measurements inaccuracy in determining the thermal conductivity coefficient doesn't exceed $7 \%$, the thermal diffusivity coefficient $-10 \%$ and the heat capacity $15 \%$.

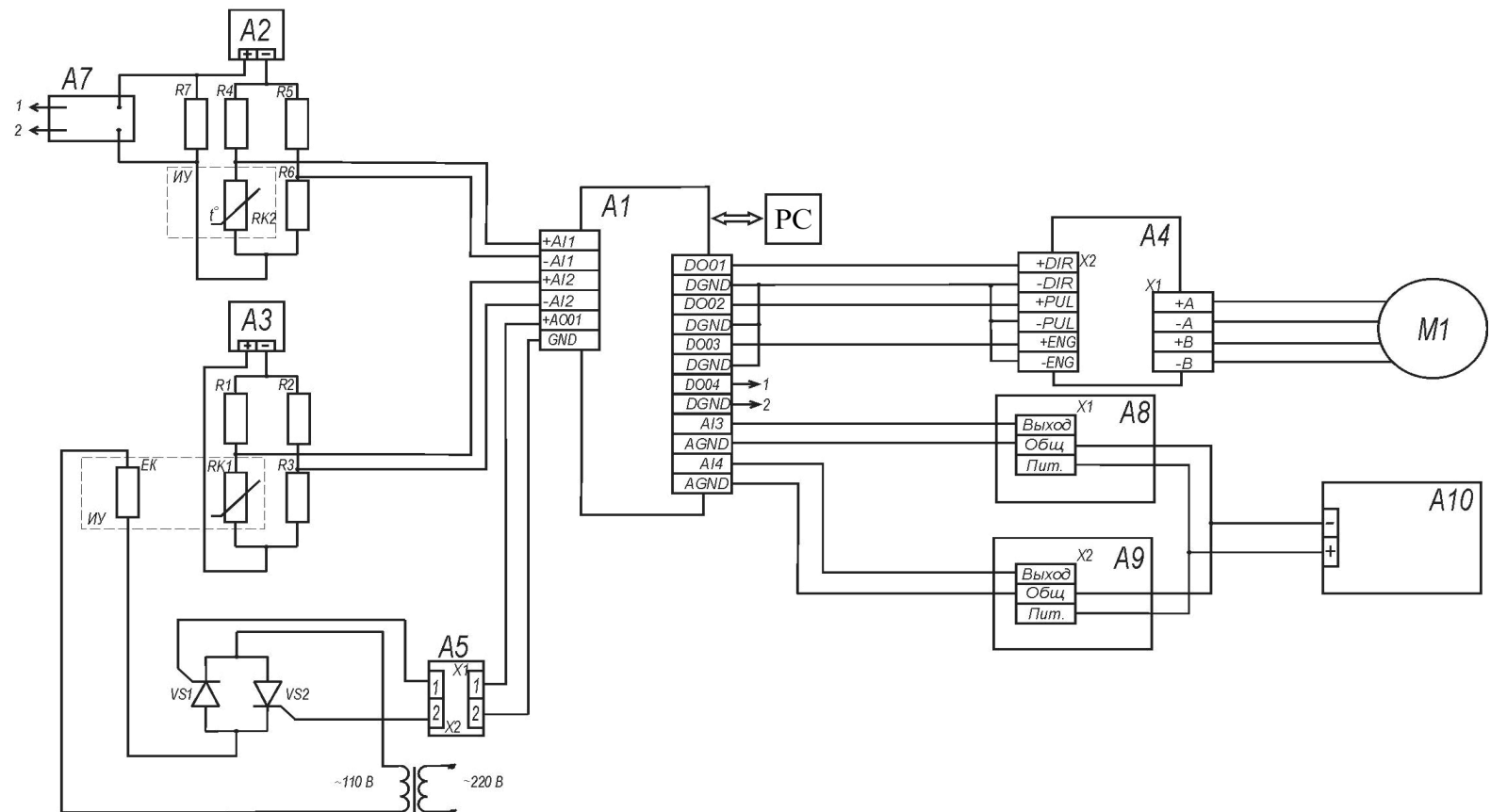

Fig. 4 - Electrical schematic diagram of the measurement setup for determining the thermo-physical characteristics of liquid depending on the sliding velocity

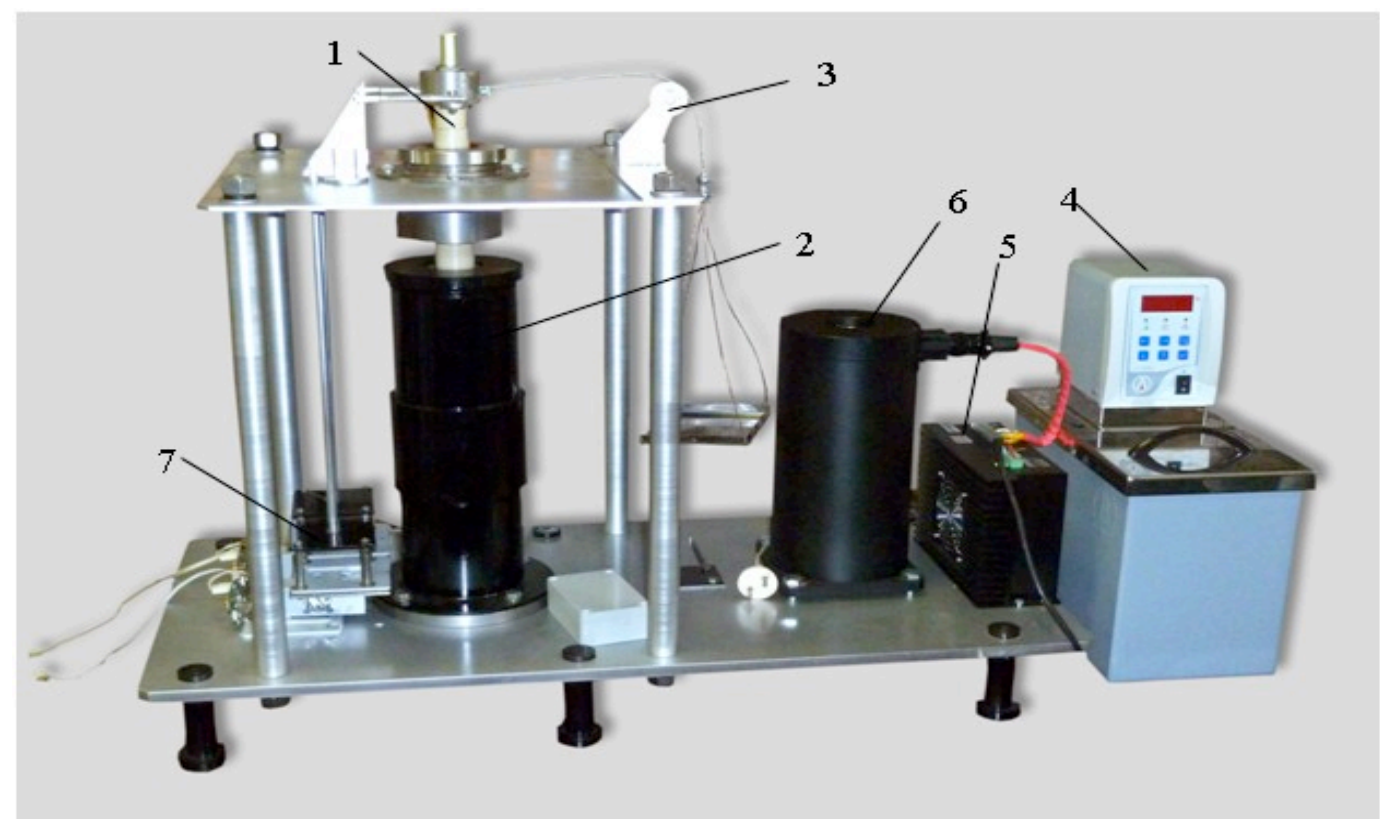


Yu.Yu. Gromov, A.A. Churikov, A.G. Divin, I.N. Ishchuk, S.A. Barkalov/

Journal of Engineering Science and Technology Review 9 (6) (2016) 135-141

Fig. 5 - the type of a measuring device for determining the dependence of the thermo-physical characteristics of liquid polymer materials on sliding velocity 1 - the inner cylinder, 2 - heat exchanger jacket, 3 - a device for the calibration of vibration frequency converters; 4 - thermostatic regulator LT-208; 5 - stepper motor control unit; 6 - stepper motor; 7 - strain gauge transducers

\section{References}

1. Ponomarev S.V., Ponomarev S.V., Mishhenko S.V., Divin A.G., Vertogradskij V.A., Churikov A.A. Theoretical and practical basis of thermo-physical measurements. Moscow, Fizmatlit, 2008.

2. Beljaev M.P., Beljaev V.P., Divin A.G. Automated system of nondestructive control over the diffusion coefficient of solvent in the products made from capillary-porous materials // Transactions of the TSTU. - 2010. - V.16. - №4. - pp.797-802.
3. Divin A.G., Ponomarev S.V., Beljaev P.S., Petrasheva M.A., Divina D.A. Method of determining thermo-physical properties of liquid polymer materials under shear flow // Transactions of the TSTU. - 2013. - V.19. - №3. - pp.500-505.

4. Lyubimova D., Divin A., Ponomarev S. Increasing the precision of thermal properties measurement by the periodic heating method // Chemical Engineering Transactions. - Vol.39. - 2014. - P. 13151320. 